\title{
Cosmic Rays and Neutrinos from GRBs: Predictions versus Acceleration Modeling
}

\author{
by D. Gialis ${ }^{1}$ and G. Pelletier ${ }^{1,2}$ \\ 1 Laboratoire d'Astrophysique de Grenoble \\ 2 Institut Universitaire de France
}

October 30, 2018

\begin{abstract}
The paper is devoted to the analysis of Fermi acceleration of protons in GRBs and its neutrino signature. We have compared the consequences of Bohm scaling and those of a Kolmogorov scaling, the latter being more reliable. The predictions about the energy limitation of UHE-protons by the various losses and the neutrino emissions turn out to be very sensitive to these scalings. We consider Kolmogorov scaling as the most realistic and predict a reasonable pp-neutrino emission around $100 \mathrm{GeV}$ at the end of the radiative stage of the fireball expansion, for a large number of GRBs pending on their baryonic load. A second $\mathrm{p} \gamma$-neutrino emission is expected with the acceleration of protons in the radiation free stage, but with a synchrotron loss limitation immediately followed by a severe expansion loss limitation. According to the Kolmogorov scaling, the protons could not reach the UHE-range. Anyway the large possibility of a two component neutrino emission would be an interesting clue of cosmic ray physics.
\end{abstract}

Keywords: GRBs, Cosmic Rays, Neutrinos, Fermi processes

\section{Introduction}

Gamma Ray Bursts (hereafter GRBs) are unique high energy phenomena in astrophysics because of their possibility to manifest all the interesting "astroparticles" processes, such as generation of high energy gamma 
rays, ultra high energy cosmic rays, high energy neutrinos and gravitational waves (see Dermer (2001) and Mészáros (2002)). The "fireball" model (Rees \& Mészáros 1992) has been successful in explaining the afterglow stage, and its more elaborated form with the addition of internal shocks (Rees \& Mészáros (1994), Paczvński \& Xu (1994)) during the "free" expansion has been successful in explaining the light curve and gamma spectra (Fishman \& Meegan (1995), Beloborodov et al. (2000)). Because of the highly relativistic dynamics necessarly involved in the GRB phenomenon, the generation of very high energy particles is expected through strong shocks and strong magnetic perturbations. Indeed GRB population could be a main source of UHE-cosmic rays which could be generated by the external shocks (Vietri 1995) or by the internal shocks (Waxman 1995), and a flux of neutrinos produced by the collisions of the UHE-cosmic rays with the GRB gamma photon is reasonably expected (Waxman \& Bahcall 1999).

In this paper, our intend is to look at the sensitivity of the predictions of cosmic ray and neutrino generation to the description of the Fermi acceleration process together with opacity effects for protons. We have analysed the consequences of two assumptions, one is the so called "Bohm scaling" assumption, the other is what we called "Kolmogorov scaling". The former consists in the statement that the Fermi acceleration time is proportional to the Larmor time of the accelerated particle, with a constant proportionality factor larger than unity (often chosen between 1 and 10 in the litterature). The latter consists in taking into account that the ratio between these two characteristic times depends on the rigidity of the particle through a law governed by the turbulence spectrum, as is confirmed by numerical works (Casse et al. 2001) , where no Bohm scaling has been found. In astrophysical media such as the solar wind, the interstellar medium, the turbulence spectrum is likely consistent with the Kolmogorov law.

The Bohm scaling is very convenient to make a first investigation of the high energy physics performances of the objects, and our prejudices are often grounded on this. However, in this paper, we will show that this scaling leads to unrealistic results. The analysis based on the Kolmogorov scaling is more reliable, and it turns out that it leads to very different conclusions, as we will present with details in the paper. The analysis is developed both in the radiation free stage and in the radiative stage. We especially emphasise the regime when the fireball is opaque to pp-collisions during the beginning of the internal shocks stage because of its baryonic load, which should occur for a large fraction of GRBs population. We make unusual predictions about 
the performance of cosmic ray generation, and about neutrino emission that could have two components, namely a non-thermal pp-neutrino emission and a $\mathrm{p} \gamma$-neutrino emission.

The paper is organised as follows. In section 2, we briefly present the dynamical description we need for the estimation of the opacity effects, not only for photons but also for protons. In section 3, we analyse the consequence of the fast acceleration regime governed by the Bohm scaling. In section 4, we present the properties of the more progressive acceleration process governed by the Kolmogorov scaling and predict a reasonable non-thermal pp-neutrino emission with its spectrum. We investigate, more briefly in section 5 , the radiation free stage, where UHE-cosmic rays are expected to be accelerated and $\mathrm{p} \gamma$-neutrinos generated. We end the paper with a discussion that summaries our conclusions about cosmic ray and neutrino generations and the sensitivity of the predictions to the acceleration model.

\section{Preliminary considerations}

\subsection{Dynamics of the fireball}

In this subsection, we summarize all the results we need for this paper that describe the expansion of the fireball (Mészáros et al. 1993). The wind flow is considered to be a set of discrete shells which are successively emitted with an energy $E_{s}=E / N_{s}$, where $N_{s}$ is the total number of shells. The duration, $t_{w}$, of this wind flow provides with an interval of shell number, namely, $1 \leq N_{s} \leq c t_{w} / r_{0}$ where $r_{0}$ is the size of the central object.

At the very beginning of the expansion of a shell, the pressure is supposed to be dominated by the radiative pressure. The temperature, $T$, of the plasma which is mainly composed by electron-positron pairs is equal to the photon temperature. Considering a shell is initially spherical with a radius, $r_{0}$, and with an energy emitted in $\gamma$-rays equal to $E_{s}$, we have

$$
\frac{E_{s}}{(4 / 3) \pi r_{0}^{3}}=a T^{4}
$$

where $a$ is the Stefan constant. Thus, the plasma temperature is

$$
T=\left(\frac{3 E_{s}}{4 \pi a r_{0}^{3}}\right)^{1 / 4} .
$$


For an energy, $E$, in the neighborhood of $10^{51}$ ergs, a number of shells, $N_{s}$, of 20 and a radius, $r_{0}$, of $10^{7} \mathrm{~cm}$, the temperature is below $10 \mathrm{MeV}$.

When a shell starts, the energy $E_{s}$ is very upper to the baryon mass energy. We can define the ratio, $\eta$, between these two energies which is

$$
\eta=\frac{E_{s}}{\left(M_{b} / N_{s}\right) c^{2}}=\frac{E}{M_{b} c^{2}} \gg 1
$$

where $M_{b}$ is the total baryonic mass ejected.

In the observer frame, the shell thickness, $\Delta r$, is supposed to remain constant and equal to $r_{0}$ until the broadening radius $r_{b}$ (Goodman (1986), Mészáros et al. (1993)). Beyond this radius, defining the Lorentz factor, $\Gamma$, of the baryonic matter, the thickness becomes

$$
\Delta r \simeq(r / c) \Delta v \simeq r / 2 \Gamma^{2}
$$

In the same frame, we also define a radius, $r_{s}$, where the kinetic energy of baryonic matter reaches its saturation value. At this moment, the Lorentz factor $\Gamma=\Gamma_{\max }$ is close to $\eta$.

In the co-moving frame, according to the Lorentz transformation, the shell thickness is given by

$$
\Delta R=\Gamma \Delta r .
$$

If we consider an adiabatic expansion of the shell, conservation of entropy in the co-moving frame for a radius lower to $r_{s}$ is given by

$$
r^{2} \Gamma r_{0} T^{3}=\text { constant }
$$

At the same time, conservation of energy is such that

$$
r^{2} \Gamma^{2} r_{0} T^{4}=\text { constant }
$$

From (21) and (3), we deduce two laws of evolution for $r \leq r_{s}$ which are $\Gamma(r) \propto r$ and $T(r) \propto r^{-1}$. Then, the saturation radius $r_{s}$ can be defined by

$$
r_{s}=\eta r_{0}
$$


For $\eta$ of the order of 300, we obtain $r_{s} \simeq 3 \times 10^{9} \mathrm{~cm}$.

Beyond the radius $r_{s}$, the equation (2) is always valid but the Lorentz factor of the shell remains constant. In this case, the temperature is such that $T(r) \propto r^{-2 / 3}$. Considering $\Delta r \geq r_{0}$, the radius $r_{b}$, according to (11), satisfies $r_{b} \geq \Gamma^{2} r_{0}$. At last, $\Gamma$ is supposed to reach its saturation value, $\eta$, around $r_{s}$ well before $r_{b}$. In fact, $\eta$ is the average value of the saturation bulk Lorentz factor, and we have to bear in mind that deviations from this average is expected to generate internal shocks. The radius $r_{b}$ is so defined by

$$
r_{b} \simeq \eta^{2} r_{0}
$$

For $\eta$ of the order of 300, we obtain $r_{b} \simeq 9 \times 10^{11} \mathrm{~cm}$.

To conclude, all the different parameters of a shell we need, can be summarized by the next following expressions :

In the observer frame, the thickness of a shell is

$$
\Delta r \simeq\left\{\begin{array}{cl}
r_{0} & \text { for } r \leq r_{b} \\
r / \Gamma_{\max }^{2} \simeq r / \eta^{2} & \text { for } r \geq r_{b}
\end{array} .\right.
$$

The Lorentz factor of a shell is such that

$$
\Gamma(r) \simeq\left\{\begin{array}{cl}
r / r_{0} & \text { for } r \leq r_{s} \\
\Gamma_{\max } \simeq \eta & \text { for } r \geq r_{s}
\end{array} .\right.
$$

In the co-moving frame, the thickness of a shell becomes equal to

$$
\Delta R \simeq\left\{\begin{array}{cl}
\Gamma r_{0} \simeq r & \text { for } r \leq r_{s} \\
\Gamma_{\max } r_{0} \simeq \eta r_{0} & \text { for } r_{s} \leq r \leq r_{b} \\
r / \Gamma_{\max } \simeq r / \eta & \text { for } r \geq r_{b}
\end{array}\right.
$$

The temperature of the pair electron-positron plasma is such that

$$
T(r) \simeq\left\{\begin{array}{cc}
10 \times\left(\frac{T\left(r_{0}\right)}{10 M e V}\right)\left(\frac{r_{0}}{r}\right) M e V & \text { for } r \leq r_{s} \\
10 \times\left(\frac{T\left(r_{0}\right)}{10 M e V}\right)\left(\frac{r_{0}}{r_{s}}\right)\left(\frac{r_{s}}{r}\right)^{2 / 3} M e V & \text { for } r \geq r_{s}
\end{array}\right.
$$


and the co-volume of a shell, $V_{c}(r)$, is

$$
V_{c}(r) \simeq\left\{\begin{array}{cl}
\Omega r^{3} & \text { for } r \leq r_{s} \\
\Omega \eta r_{0} r^{2} & \text { for } r_{s} \leq r \leq r_{b} \\
(\Omega / \eta) r^{3} & \text { for } r \geq r_{b}
\end{array}\right.
$$

where $\Omega(\simeq 4 \pi / 500)$ is the opening angle of the emission.

The collision date (if any...), $t_{c}$, between two shells of Lorentz factor $\gamma_{1}$ and $\gamma_{2}$, which started at two different times separated by $\Delta t_{0}$ is such that $t_{c} \simeq 2 \frac{\gamma_{1}^{2} \gamma_{2}^{2}}{\left|\gamma_{1}^{2}-\gamma_{2}^{2}\right|} \Delta t_{0}$, which leads to collisions at various distances $r_{c}$ such that $r_{c} / r_{b} \sim \Delta t_{0} / t_{0}$ (see Daigne \& Mochkovitch (1998)). For a long GRB having a duration $t_{w} \sim 10 \mathrm{~s}$, the maximum collision radius reaches the deceleration radius $r_{d}$. The light curve duration could be due to either the duration of the wind $t_{w}$ or to the time spread of the shell at the most remote collision, namely, $\Delta t_{d}=t_{d} / \eta^{2}$. It turns out that this latter time is comparable to the duration of the wind of a long GRB, namely, $1-10 \mathrm{~s}$. The observed millisecond variations should come from internal collisions located at a few $r_{b}$ where $\Delta r_{b} \sim r_{0}$.

Anyway after a few $r_{b}$, all the shells mix up and form a single jet. That is the reason why volume and thickness of a shell can really be defined only before the broadening radius. After this radius, in the equations (4) and (5), they are undervalued.

\subsection{Conversion of the GRB energy into cosmic rays}

During the evolution, the energy of the fireball is shared between several forms : the thermal energy $E_{t h}$, the magnetic energy $E_{m}$, the bulk kinetic energy, the cosmic ray energy $E_{\star}$ and the energy in the form of hydromagnetic perturbations $E_{m}^{\star}$. The energy share $E_{m}^{\star}$ is the reservoir for particle acceleration.

Until the saturation radius $r_{s}$, the ratio of the pair thermal energy over the fireball energy, $\left(a T^{4} V_{c}\right) / E_{s}$, starts with a value close to unity and then decays as $1 / r$. In this stage, the proton population is a tiny contribution both in number of particles and in energy. Assuming that the protons dominate the baryonic load, we get the total number of protons

$$
N_{p} \simeq \frac{M_{b}}{m_{p}} \simeq 0.67 \times 10^{51}\left(\frac{\eta}{300}\right)^{-1}\left(\frac{E}{10^{51} \mathrm{erg}}\right)
$$


Thus, the density of protons by shell, $n_{p}(r)$, in the co-moving frame and before the broadening radius, is such that

$$
n_{p}(r) \geq 10^{-4}\left(\frac{t_{0}}{1 m s}\right)\left(\frac{t_{w}}{10 s}\right)^{-1} \frac{N_{p}}{V_{c}(r)},
$$

and the maximum value of $n_{p}(r)$ is equal to $N_{p} / V_{c}(r)$.

For $t_{0}=r_{0} / c=1 \mathrm{~ms}$, we can write

$n_{p}(r) \geq\left\{\begin{array}{l}2.7 \times 10^{27}\left(\frac{t_{w}}{10 s}\right)^{-1}\left(\frac{\eta}{300}\right)^{-1}\left(\frac{\Omega / 4 \pi}{2 \times 10^{-3}}\right)^{-1}\left(\frac{E}{10^{51} \text { erg }}\right)\left(\frac{r}{r_{0}}\right)^{-3} c m^{-3} \\ 1.0 \times 10^{20}\left(\frac{t_{w}}{10 s}\right)^{-1}\left(\frac{\eta}{300}\right)^{-2}\left(\frac{\Omega / 4 \pi}{2 \times 10^{-3}}\right)^{-1}\left(\frac{E}{10^{51} \text { erg }}\right)\left(\frac{r}{r_{s}}\right)^{-2} c^{-3}\end{array}\right.$

respectively for $r \leq r_{s}$ and $r_{s} \leq r \leq r_{b}$.

Considering $\eta=300$, even if the GRB has a long duration about $10 \mathrm{~s}$, the density of protons, at $r=r_{s}$, is larger than $10^{20} \mathrm{~cm}^{-3}$. We note that, up to the saturation radius, the density of a shell is comparable to the density of a solid.

The corresponding ratio of the thermal energy of protons, $3 T /\left(2 \eta m_{p} c^{2}\right)$, starts with a very low value $\left(\sim 10^{-4}\right)$ and decays like $1 / r$ as well. The cosmic ray component is supposed to develope out of the proton thermal component and would grow up to several percents of $E$ to account for a significant contribution of the UHE cosmic ray generation in the Universe. Assume that a fraction $\xi_{\star}$ of the proton population is injected into the cosmic ray component (i.e. $N_{\star}=\xi_{\star} N_{p}$ ) and that they reach a mean energy $\bar{\epsilon}(r)=$ $\bar{\gamma}(r) m_{p} c^{2}$ in the co-moving frame. Then, the contribution to the energy of the fireball, in the observer frame, is given by

$$
E_{\star}(r)=\frac{\xi_{\star} \bar{\gamma}(r) \Gamma(r)}{\eta} E
$$

When the radius $r$ reaches the saturation radius $r_{s}$, the energy of the cosmic ray component is equal to $\xi_{\star} \bar{\gamma}(r) E /\left(1+4 \xi_{\star} \bar{\gamma}(r) / 3\right)$. For a cosmic ray spectrum in $\epsilon^{-2}, \bar{\gamma}=\log \left(\gamma_{\max }\right)$, which clearly shows that the goal of converting about 10 percents of the fireball energy into cosmic rays energy is achieved when a sizeable fraction of the protons are injected in the cosmic ray population.

Regarding the magnetic energy of the fireball, two points of view can be considered; either the magnetic field behaves like in a jet and the poloidal 
component decreases like $1 / r^{2}$ thus its pressure decreases like the relativistic thermal pressure in $1 / r^{4}$; whereas the toroidal component decreases more slowly; or the magnetic field of the shell disconnects from the central source; which is very likely. In this latter case, the magnetic energy of the shell is conserved as long as the dissipation is negligible (e.g. $B \propto V_{c}^{-1 / 2}$ ) and decays when the Fermi processes and/or reconnections become efficient in accelerating particles. Initially, the intensity of magnetic field is usually considered as of the order of the equipartition value, which means that the magnetic energy is a sizeable fraction of the fireball energy $\left(E_{m}\left(r_{0}\right)=\xi_{m} E\right)$.

\subsection{The importance of pp-collisions during the primeval stage}

We begin with defining the radius $r_{\star}$ where a shell becomes optically thin with respect to Compton scattering. It can easily be checked that a typical shell width $\Delta R$ becomes smaller than the flow transverse radius after a short while, when $r>\eta \sqrt{\pi / 4 \Omega} r_{0}$ which is comparable to $r_{s}$. It will turn out that the photosphere is located at a much larger distance for large enough $\eta$ (see

(6) ) and therefore the opacity of a shell is determined by its width. The Compton opacity is $\tau_{\star}=\sigma_{T} n_{e} \Delta R$ where $\sigma_{T}$ is the Thompson cross section. We assume $n_{e} \simeq n_{p} \simeq n_{b}$ and thus the co-moving baryon density is related to the wind mass flux $\dot{M}_{w}$ by

$$
n_{b}=\frac{\dot{M}_{w}}{\Gamma \Omega r^{2} m_{p} c}
$$

Moreover for $r>r_{s}$, the GRB energy is converted into relativistic kinetic energy such that the kinetic luminosity (kinetic energy flux) of the wind $L_{w}=E / t_{w}=\eta \dot{M}_{w} c^{2}$. For $r>r_{s}, \Gamma \simeq \eta$ and the Compton opacity is thus

$$
\tau_{\star}=\frac{\sigma_{T} L_{w}}{\Omega m_{p} c^{3} \eta^{2}} \frac{\Delta R}{r^{2}} .
$$

We can define a critical value for $\eta$ such that the photospheric radius is located at $r_{b}$, where shock acceleration starts. This critical value $\eta_{\star}$ is given by

$$
\eta_{\star} \equiv\left(\frac{\sigma_{T} L_{w}}{\Omega m_{p} c^{3} r_{0}}\right)^{1 / 5} \simeq 570 \times\left(\frac{E}{10^{51} e r g}\right)^{1 / 5}\left(\frac{t_{w}}{1 s}\right)^{-1 / 5}\left(\frac{\Omega / 4 \pi}{2 \times 10^{-3}}\right)^{-1 / 5}
$$


The photospheric radius is such that

$$
r_{\star}=\left\{\begin{array}{cc}
r_{b}\left(\eta_{\star} / \eta\right)^{5} & \text { for } \eta \leq \eta_{\star} \\
r_{b}\left(\eta_{\star} / \eta\right)^{5 / 2} & \text { for } \eta \geq \eta_{\star}
\end{array} .\right.
$$

These simple formulae illustrate the requirement of a large value of the asymptotic bulk Lorentz factor $\eta$ in order to observe an optically thin X-ray spectrum. For $\eta$ larger than the critical value, the internal shocks generate only a non-thermal spectrum just after $r_{b}$, whereas, for $\eta<\eta_{\star}$, the internal shocks start accelerating particles in an optically thick plasma. Assuming that the GRB energy varies within two orders of magnitude, the wind time by two orders of magnitude and the solid angle by one order of magnitude, the possible values of $\eta_{\star}$ extend within an interval of one order of magnitude. However it is worth mentioning that this usual approach of the opacity issue is an underestimate of the opacity effects, because the coalescence of shells and ultimately their dissolution into a single jet increase the opacity.

The possibility for the internal shock scenario to partially operate in an optically thick regime is important when one consider pp-collisions. Indeed, any relativistic protons can collide with other protons (or neutrons) producing pions and thus neutrinos. The cross section, $\sigma_{p p}$, for this production is constant and equal to $2.7 \times 10^{-26} \mathrm{~cm}^{2}$ when the kinetic energy of protons is larger than $1 \mathrm{GeV}$. The sheets become thin to pp-collisions always before the photosphere since $\tau_{p p}=n_{p} \sigma_{p p} \Delta R=1$ at $r_{p p}$ such that $r_{p p}=r_{\star} \sqrt{\sigma_{p p} / \sigma_{T}}<r_{\star}$ for $r_{p p}<r_{b}$ and $r_{p p}=r_{\star} \sigma_{p p} / \sigma_{T}<r_{\star}$ for $r_{p p}>r_{b}$. In this latter case, $r_{p p} \simeq 0.04 r_{\star}$; which occurs when $\eta \leq \eta_{\star} / 2$. In this paper, we intend to emphasize the importance of this energy limitation to proton acceleration and estimate the resulting neutrino emission.

\section{Consequences of a fast acceleration regime}

In this section, we analyse the consequences of the usual assumption of a Bohm scaling for the Fermi process for both the energy distribution cut off and the depletion of the energy reservoir for acceleration. 


\subsection{Bohm scaling and energy losses}

We first consider a Fermi acceleration process that is assumed to follow the so called Bohm's scaling namely characterized by its time scale proportional to the Larmor time, i.e. :

$$
t_{a c c}=\kappa_{0} t_{L}=\kappa_{0} \frac{10^{-4}}{Z}\left(\frac{\epsilon}{1 G e V}\right)\left(\frac{B}{1 G}\right)^{-1} s,
$$

where we take $\kappa_{0}=10$, as often assumed in astroparticle physics.

In the early phase, the cut off of the proton energy distribution can be caused by either the synchrotron loss or the pp-collisions; which is obtained by equating the acceleration time and the loss time. The synchrotron time $t_{\text {syn }}$ is given by

$$
t_{\text {syn }}^{-1}=\frac{4 Z^{4} \sigma_{T} \gamma}{3 m c}\left(\frac{m_{e}}{m}\right)^{2} W_{m}
$$

where $W_{m}$ is the density of magnetic energy.

This expression leads to the cut off energy :

$$
\epsilon_{\text {syn }} \simeq 2.4 \times 10^{11} \frac{1}{\sqrt{\kappa_{0}}}\left(\frac{B}{1 G}\right)^{-1 / 2} \mathrm{GeV} .
$$

We consider now the limitation caused by pp-collisions. The cut off energy $\epsilon_{p p}$ is such that $\left(n_{p} \sigma_{p p} c\right) \kappa_{0} t_{L}=1$, and thus

$$
\epsilon_{p p} \simeq \frac{10^{4}}{\kappa_{0}}\left(\frac{B}{1 G}\right)\left(\frac{\eta^{6}}{\eta_{\star}^{5}}\right)\left(\frac{r_{0}}{c}\right) G e V .
$$

At the broadening radius $\left(r_{b}\right)$, where the magnetic field could be as high as $10^{7} \mathrm{G}$, these two limitations are comparable :

$$
\epsilon_{\text {syn }} \simeq 2.4 \times 10^{7}\left(\frac{\kappa_{0}}{10}\right)^{-1 / 2}\left(\frac{B}{10^{7} G}\right)^{-1 / 2} \mathrm{GeV},
$$

and

$$
\epsilon_{p p} \simeq 3 \times 10^{9}\left(\frac{\kappa_{0}}{10}\right)^{-1}\left(\frac{B}{10^{7} G}\right)\left(\frac{\eta}{300}\right)\left(\frac{\eta}{\eta_{*}}\right)^{5} \mathrm{GeV}
$$


During the expansion the ratio between these two limitations evolves such that

$$
\frac{\epsilon_{p p}}{\epsilon_{s y n}} \simeq 1.2 \times 10^{3}\left(\frac{\kappa_{0}}{10}\right)^{-1 / 2}\left(\frac{\eta}{300}\right)\left(\frac{\eta}{\eta_{*}}\right)^{5}\left(\frac{B}{10^{7} G}\right)^{3 / 2}\left(\frac{r}{r_{b}}\right)^{2}
$$

which suggests that for any decrease of the magnetic field in $r^{-\alpha}$ with $\alpha$ between 1 and 2, the most severe limitation would be due to synchrotron losses. However we will show in the next subsection that such efficient Fermi acceleration would dissipate the magnetic energy very rapidly.

\subsection{The depletion of the energy reservoir for acceler- ation}

Such an efficient acceleration produces a strong depletion of the energy reservoir for particle acceleration. The previous results would make sense only if the depletion of the energy reservoir for particle acceleration is slow. This is exactly the purpose of this subsection.

Let $E_{m}^{\star}$ be the energy in the hydromagnetic perturbations (magnetic and kinetic energy) involved in the acceleration of particles. We assume that this is a sizeable fraction $\xi_{m}^{\star}$ of the total magnetic energy and that even the large scale magnetic field in the shell is mostly disconnected from the central source after a short while and thus is rather tangle. Therefore we assume that the total magnetic energy dissipates at the same rate than $E_{m}^{\star}$. A minimum dissipation is assumed by considering its energy loss by particle acceleration only. According to (3.1), the acceleration power is $Q^{+}=\frac{N_{\star}}{\kappa_{0} t_{L 0}^{\prime}} m_{p} c^{2}$, where $t_{L 0}^{\prime}$ is the Larmor time for a proton of $1 \mathrm{GeV}$ in the co-moving frame. The minimum depletion is then governed by $\dot{E}_{m}^{\star}=-Q^{+}$, which reads :

$$
\dot{E}_{m}=-\frac{E}{t_{\star}^{\prime}} \frac{\bar{B}}{B_{\star}}
$$

where we define a characteristic time $t_{\star}^{\prime}$ at a given radius $\tilde{r}$ where the magnetic field has an intensity $B_{\star}$, such that

$$
t_{\star}^{\prime} \equiv\left(\eta \kappa_{0} \xi_{m}^{\star} / \xi_{\star}\right) t_{L 0}^{\prime}\left(B_{\star}\right) .
$$

Clearly, this time measures the rate of depletion at the considered radius $\tilde{r}$ and must be compared to the co-moving dynamical time $t^{\prime}(\tilde{r})=\tilde{r} / \Gamma c$. Let us 
make an estimate at $r_{b}$, the late stage actually; for $B=10^{7} \mathrm{G}$ and $\epsilon=1 \mathrm{GeV}$, $t_{\star}^{\prime} \simeq 3 \times 10^{-8} \mathrm{~s}$, whereas $t^{\prime}\left(r_{b}\right) \simeq 10^{-2} \mathrm{~s}$. This perturbation burning out within a few nanoseconds is not realistic at all. Before this stage, the depletion time is much shorter than the fireball dynamical time by a factor proportional to $(B r)^{-1}$ that goes at best like $r^{1 / 2}$ for $B \propto r^{-3 / 2}$ corresponding to magnetic energy conservation and the perturbations are burnt out almost immediately, their energy being radiated by photons and neutrinos. The observation of these perturbations in the light curve of gamma emissions suggests a much slower depletion; which we will analyse in the next section.

\section{The interest of a progressive acceleration regime}

The Bohm scaling is often used in astroparticle physics, which sometimes can provide some rough estimate of the high energy cut off. But the true scaling (Casse et al. 2001) depends on the turbulence spectrum. We will assume that the perturbations are distributed according to the Kolmogorov law. This will significantly change the performance of the Fermi process and the conclusions about cosmic ray generation.

\subsection{Kolmogorov scaling and energy losses}

In the co-moving frame, the acceleration time depends on the speed $\beta_{\star} c$ of magnetic perturbations that scatter particles on both sides of the shocks. Considering the same average velocity, we have the relation

$$
t_{a c c}=\frac{t_{s}}{\beta_{\star}^{2}}
$$

where the scattering time, $t_{s}$, can be expressed like

$$
t_{s}=\left(\eta_{b} \rho^{\beta-1} \omega_{L}\right)^{-1}
$$

with $\eta_{b}=\frac{<\delta B^{2}>}{\bar{B}^{2}}$ and $\rho=\frac{r_{L}}{l_{c}} \leq 1$ where $l_{c}$ is the mean coherence length of the magnetic perturbations whose spectrum is supposed to be a power law of index $\beta$. According to the Kolmogorov theory, one can take $\beta=5 / 3$ and, with $\kappa_{0}=1 /\left(\beta_{\star}^{2} \eta_{b}\right)$, we get

$$
\kappa=\kappa_{0} \rho^{-2 / 3} .
$$


Considering $l_{c} \lesssim \Delta R$,

$$
\rho=\frac{r_{L}}{\Delta R} \simeq \frac{\epsilon}{Z e B \Delta R c}
$$

and we have

$$
\kappa \simeq 2.2 \times 10^{-3}\left(\frac{\kappa_{0}}{10}\right)\left(\frac{\epsilon}{1 G e V}\right)^{-2 / 3}\left(\frac{B}{1 G}\right)^{2 / 3}\left(\frac{\Delta R}{1 \mathrm{~cm}}\right)^{2 / 3} .
$$

The synchrotron limitation becomes

$$
\epsilon_{\text {syn }} \simeq 1.2 \times 10^{19}\left(\frac{\kappa_{0}}{10}\right)^{-3 / 4}\left(\frac{B}{1 G}\right)^{-5 / 4}\left(\frac{\Delta R}{1 \mathrm{~cm}}\right)^{-1 / 2} \mathrm{GeV} .
$$

For $r=r_{b}\left(\simeq 10^{12} \mathrm{~cm}\right), B=10^{7} \mathrm{G}$ and $\Omega=4 \pi / 500, \epsilon_{s y n} \simeq 3.8 \times 10^{5} \mathrm{GeV}$.

As for the pp-collision limitation,

$$
\epsilon_{p p} \simeq\left(\frac{\kappa_{0}}{10}\right)^{-3}\left(\frac{\eta}{300}\right)^{3}\left(\frac{\eta}{\eta_{\star}}\right)^{15}\left(\frac{B}{10^{7} G}\right)\left(\frac{\Delta R}{10^{12} \mathrm{~cm}}\right)^{-2}\left(\frac{r}{r_{b}}\right)^{6} \mathrm{GeV} .
$$

For $r \geq r_{b}, \Delta R=r / \eta$, defining $r_{b \star} \equiv \eta_{\star}^{2} r_{0}$, we obtain

$$
\epsilon_{p p} \simeq 2.0 \times 10^{5}\left(\frac{\kappa_{0}}{10}\right)^{-3}\left(\frac{B}{10^{7} G}\right)\left(\frac{\eta_{\star}}{570}\right)\left(\frac{\eta}{\eta_{\star}}\right)^{8}\left(\frac{r}{r_{b \star}}\right)^{4} G e V .
$$

This energy increases up to a maximum value reached at $r_{p p}$ (when it's larger than $r_{b}$ ), namely,

$$
\epsilon_{p p} \simeq 3.5 \times 10^{2}\left(\frac{\kappa_{0}}{10}\right)^{-3}\left(\frac{B}{10^{7} G}\right)\left(\frac{\eta_{\star}}{570}\right)\left(\frac{\eta_{\star}}{\eta}\right)^{4} \mathrm{GeV} .
$$

As long as $r<r_{p p}$, the energy limitation is clearly due to pp-collisions rather than synchrotron losses. These results show, first, that it is not possible to accelerate protons beyond $100 \mathrm{GeV}$ before the fireball becomes thin for protons at $r_{p p}$, for $\eta>\eta_{\star}$; second, that, for $\eta<\eta_{\star} / 2$, the proton energy could increase above $100 \mathrm{GeV}$. For instance, if $\eta=\eta_{\star} / 3, r_{\star} \simeq 240 r_{b}, r_{p p} \simeq 10 r_{b}$, $B$ weakens by a factor $\left(r_{p p} / r_{b}\right)^{-\alpha} \simeq 3.2 \times 10^{-2}$ with $\alpha=3 / 2$ and thus compensates the increase due to $\eta$. Therefore the energy remains of order 100 $\mathrm{GeV}$. We have to examine how this regime changes the rate of perturbation energy depletion.

In the opposite situation where the protons are not accelerated beyond $\mathrm{GeV}$ in the opaque stage, for $\eta>\eta_{\star}$ and/or $B<10^{5} \mathrm{G}$ at $r_{b}$, nucleosynthesis is possible as shown by Lemoine (Lemoine 2002). 


\subsection{The depletion of the energy reservoir for acceler- ation}

Taking into account the variation of $\kappa$ in the acceleration power, we define a new characteristic time $t_{\star}^{\prime}$ which is quite different from the last one :

$$
t_{\star}^{\prime} \equiv\left(\eta \kappa_{0}<\rho^{2 / 3}>^{-1} \xi_{m}^{\star} / \xi_{\star}\right) t_{L 0}^{\prime}\left(B_{\star}\right)
$$

For a power law distribution, not harder than $\epsilon^{-2},<\rho^{2 / 3}>\sim \rho_{0}^{2 / 3}, \rho_{0}$ being the rigidity for $1 \mathrm{GeV}$. For bell type distribution of standard deviation $\bar{\epsilon}$, $<\rho^{2 / 3}>\sim<\rho(\bar{\epsilon})^{2 / 3}>$. Compared to the Bohm regime, the Kolmogorov regime increases this time by a factor $\rho_{0}^{-2 / 3}$. At $r_{b}$ we get a more extended time of the order of $0.1 \mathrm{~s}$, which is much more realistic than in the Bohm scaling case. If $r_{p p}$ is significantly larger, the depletion behaviour is changed such that $t_{\star}^{\prime} / t^{\prime}$ decreases instead of increasing like in Bohm regime. Indeed in Kolmogorov regime, this ratio scales like $B^{-1 / 3} r^{-5 / 9}$, which decreases when $B$ decreases less slowly than $r^{-5 / 3}$.

For a more detailed estimate, we integrated the differential equation (17) which, after some algebra, leads to the following result :

$$
\frac{E_{m}}{E_{m}\left(r_{b}\right)}=\left[1-\frac{E}{E_{m}\left(r_{b}\right)} \frac{t^{\prime}\left(r_{b}\right)}{5 t_{\star}^{\prime}\left(r_{b}\right)}\left(1-\left(\frac{r}{r_{b}}\right)^{-5 / 6}\right)\right]^{6} .
$$

In fact, the perturbations are burnt out in a fraction $\delta t^{\prime}$ of $t_{\star}^{\prime}\left(r_{b}\right)$, which can be obtained directly from the differential equation (7) :

$$
\delta t^{\prime}=t_{\star}^{\prime}\left(r_{b}\right) \frac{E_{m}\left(r_{b}\right)}{E},
$$

which confirms the previous statement of a reasonable depletion time.

\subsection{The pp-neutrino emission}

In this subsection, we give an evaluation of the number of emitted ppneutrinos and we calculate their energy spectrum. 
The pp-collisions produce neutrinos after some reactions which are

$$
\begin{array}{cc}
p+p \longrightarrow c & D+\pi^{+} \\
p+p+a\left(\pi^{+}+\pi^{-}\right)+b \pi^{0} \\
p+n+\pi^{+}+a\left(\pi^{+}+\pi^{-}\right)+b \pi^{0} \\
2 n+2 \pi^{+}+a\left(\pi^{+}+\pi^{-}\right)+b \pi^{0}
\end{array}
$$

The production of mesons $\pi^{-}$and $\pi^{+}$gives neutrinos et $\mu$-mesons through the decay reactions

$$
\begin{aligned}
& \pi^{-} \longrightarrow \mu^{-}+\bar{\nu}_{\mu} \\
& \pi^{+} \longrightarrow \mu^{+}+\nu_{\mu}
\end{aligned}
$$

and other neutrinos are produced after

$$
\begin{array}{r}
\mu^{-} \longrightarrow e^{-}+\bar{\nu}_{e}+\nu_{\mu} \\
\mu^{+} \longrightarrow e^{+}+\nu_{e}+\bar{\nu}_{\mu} .
\end{array}
$$

Let's write energy of different particles

$$
\begin{aligned}
m_{\pi^{ \pm}} & \simeq 140 \mathrm{MeV} / \mathrm{c}^{2} \\
m_{\mu} & \simeq 105 \mathrm{MeV} / \mathrm{c}^{2} . \\
m_{e^{ \pm}} & \simeq 0.5 \mathrm{MeV} / \mathrm{c}^{2}
\end{aligned}
$$

Thus, neutrinos coming from (11) have a minimum energy equal to

$$
\epsilon_{\nu_{\mu}} \simeq \frac{m_{\pi^{ \pm}}^{2}-m_{\mu}^{2}}{2 m_{\pi^{ \pm}}} \simeq 30 \mathrm{MeV}
$$

and decay of $\mu$-mesons (12) gives neutrinos having a minimum energy which varies from 25 to $50 \mathrm{MeV}$.

Let $f_{\nu}\left(\epsilon_{\nu}\right)$ be the energy distribution of neutrinos, normalised such that $\int f_{\nu}\left(\epsilon_{\nu}\right) d \epsilon_{\nu}=n_{\nu}$, the number density of neutrinos; and $f_{\star}(\gamma)$ the Lorentz factor distribution of relativistic protons, normalised such that $\int f_{\star}(\gamma) d \gamma=n_{\star}$, the number density of relativistic protons. The kinetic equation for neutrinos can be written in a simplified way as follows :

$$
\frac{\partial}{\partial t} f_{\nu}+c \vec{n} . \nabla f_{\nu}=\xi_{\nu} \nu_{p p} \int f_{\star}(\gamma) \delta\left(\epsilon_{\nu}-\gamma_{c} \epsilon_{0}\right) d \gamma
$$


where $\epsilon_{0}$ is the average energy of the neutrinos generated by the pp-collision in the center of mass frame (above estimated), $\xi_{\nu}$ the average number of produced neutrinos at each collision and $\gamma_{c}$ the Lorentz factor of the collision frame. The energy of the emitted neutrinos remain close to the minimum values because the pions do not take all the energy available above the threshold, but just a little excess above it, the remaining energy being kept by the proton or the neutron. Therefore the number $\xi_{\nu}$ is just a few. The Lorentz factor $\gamma_{c} \sim \sqrt{\gamma}$, where $\gamma$ is the Lorentz factor of the relativistic proton. So we can easily deduce that, for $f_{\star} \propto \gamma^{-s}$, the energy flux of neutrinos is in $\epsilon_{\nu}^{-2 s+2}$, between $\epsilon_{0}$ and $\epsilon_{0} \sqrt{\epsilon_{p p} / m_{p} c^{2}}$, in the co-moving frame. For $s=2$, a neutrino spectrum in $\epsilon_{\nu}^{-2}$ is expected between $5 \mathrm{GeV}$ and $150 \mathrm{GeV}$, typically, for the observer frame seing the GRB flow coming front.

Assuming an isotropic neutrino emission in the co-moving frame, the power emitted is

$$
\dot{E}_{\nu}^{\prime}=\Omega \xi_{\nu} \epsilon_{0} \int \nu_{p p} n_{\star} r^{2} d(\Delta R)
$$

where the integral over the width extends down to the lower bound at $r_{p p}$, corresponding to $\tau_{p p}=1$. Thus $E_{\nu}^{\prime}=t_{w}^{\prime} \Omega \xi_{\nu} \epsilon_{0} c n_{\star} r_{p p}^{2}$. The quantity $t_{w}^{\prime} \Omega c n_{\star} r_{p p}^{2}$ is the number of relativistic protons $\xi_{\star} N_{p}$ which have flown through the "proto"-sphere during the GRB event. Finally, we get a simple formula giving the amount of neutrino energy in the co-moving frame :

$$
E_{\nu}^{\prime}=\xi_{\star} \xi_{\nu} \frac{\epsilon_{0}}{m_{p} c^{2}} \frac{E}{\eta} .
$$

For an observer seing the GRB shells coming front, the neutrino energy emission is multiplied by the bulk Lorentz factor, thus $E_{\nu}=\xi_{\star} \xi_{\nu}\left(\epsilon_{0} / m_{p} c^{2}\right) E$ which reasonably leads to $E_{\nu} \sim 10^{-3}-10^{-2} E$. The number of emitted neutrinos by a GRB is simply $\xi_{\nu} N_{\star}$. A neutrino telescope of collecting surface $A$ can receive $N_{\nu} A / 4 \pi D^{2}$ from a GRB exploding at a distance $D$. For example, a GRB occuring at $100 \mathrm{Mpc}$ can provide with $10^{5}$ neutrinos crossing a $\mathrm{km}^{2}$-detector. However, the number of events recorded by the detector is obtained by multiplying this number by the detection probability which is quite low.

In the same process, $\pi^{0}$-decay occurs and generates gamma photons. An excess of these photons could be seen in the gamma spectrum; they superimpose a spectrum in $\epsilon_{\gamma}^{-2 s+2}$ on the synchrotron spectrum due to the electrons. 
Because we focused on proton acceleration and neutrino emission, we disregarded the interesting issue of the neutron component which can decouple under some conditions (Derishev et al. 1999).

\section{The stage of UHE Cosmic Rays accelera- tion}

Because of their magnetic field intensity and the size of their wind, the GRBs are considered as possible accelerators of UHE-cosmic rays Vietri (1995), Waxman (1995)) : for $r>r_{b}$, particles could reach the maximal energy beyond which they are no longer confined, $\epsilon_{\max }=Z$ e $B r$, namely

$$
\epsilon_{\max }=7.7 \times 10^{21}\left(\frac{B}{10^{7} G}\right)\left(\frac{r}{10^{12} \mathrm{~cm}}\right) \mathrm{eV},
$$

for a proton $(Z=1)$.

\subsection{Proton energy in the transparent stage}

We examine the loss limitation suffered by the protons after crossing the "proto"-sphere. After the equation (8), we have already given the value of the maximum energy due to the synchrotron loss at $r_{b}$; thus, it scales like :

$$
\epsilon_{\text {syn }} \simeq 3.8 \times 10^{5}\left(\frac{B\left(r_{b}\right)}{10^{7} G}\right)^{-5 / 4}\left(\frac{r}{r_{b}}\right)^{(5 \alpha-2) / 4} \mathrm{GeV} .
$$

It will turn out that this value is above the threshold of the p $\gamma$-process, but is far below the range expected for getting UHE-Cosmic Rays. For $\alpha=3 / 2$, this range would be reached at a few $100 r_{b}$.

The expansion loss can be significant. Indeed, the cut off energy is obtained by setting $t_{a c c}=t^{\prime}=t / \Gamma$ in the co-moving frame, and the limitation, $\epsilon_{\text {exp }}$, is such that

$$
\epsilon_{e x p} \simeq 10^{5}\left(\frac{\kappa_{0}}{10}\right)^{-3}\left(\frac{\eta}{300}\right)\left(\frac{B\left(r_{b}\right)}{10^{7} G}\right)\left(\frac{r}{r_{b}}\right)^{1-\alpha} \mathrm{GeV} .
$$

Whereas the synchrotron loss diminishes with distance, the expansion limitation becomes more and more severe (see figure 10. Moreover, it can easily 
be checked that the escape time due to transverse diffusion is always much longer than the expansion time.

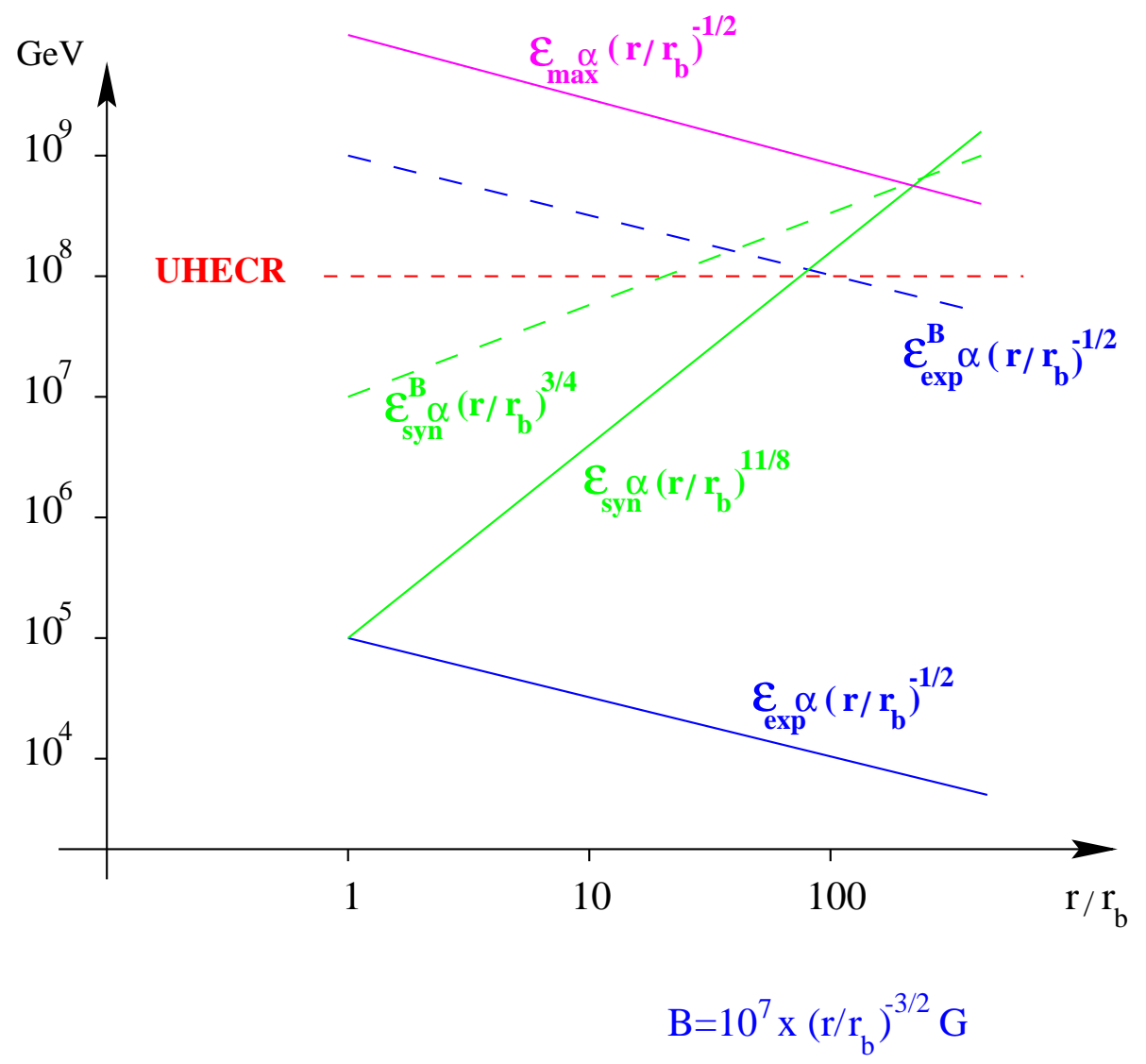

Figure 1: Diagram of energy limitation in the co-moving frame for $\alpha=3 / 2$ : the dashed lines show the result of the Bohm scaling and the solid lines are obtained with the Kolmogorov scaling. The horizontal dashed line represents the lowest UHECR range limit.

\subsection{The $\mathrm{p} \gamma$-neutrino emission}

A signature of VHE-protons acceleration is expected with the neutrino production resulting from the photo-production of pions, which is efficient to 
produce neutrinos through the $\Delta-$ resonance (the so-called GZK effect) :

$$
\begin{aligned}
& p+\gamma \rightarrow \Delta^{+} \stackrel{(2 / 3)}{\rightarrow} p+\pi^{0} \rightarrow p+\gamma+\gamma \\
& \stackrel{(1 / 3)}{\rightarrow} n+\pi^{+} \rightarrow \ldots \rightarrow p+e^{+}+e^{-}+\nu_{e}+\bar{\nu}_{e}+\nu_{\mu}+\bar{\nu}_{\mu}
\end{aligned}
$$

The threshold of the process is such that $\epsilon_{p} \epsilon_{\gamma} \geq \frac{1}{2} m_{n} m_{\pi}-\frac{1}{4} m_{\pi}^{2}$, which leads to a threshold for the proton Lorentz factor $\gamma_{t h} \sim 10^{4}$, the energy of the photons being in the $\mathrm{keV}$ range in the co-moving frame. As previously seen, $\gamma_{\max }$ is at least ten times larger than $\gamma_{t h}$. The GRBs are likely opaque to this process until the shells reach the radius $r_{p \gamma}$ such that

$$
r_{p \gamma}=\left(\frac{\sigma_{p \gamma} E_{\gamma}}{\Omega \bar{\epsilon}_{\gamma}}\right)^{1 / 2}
$$

where $\bar{\epsilon}_{\gamma}$ is the average energy of the target photons in the observer frame (typically $1 \mathrm{MeV}$ ); which puts this p $\gamma$-" proto"-sphere at a rather large radius of $1.5 \times 10^{15} \mathrm{~cm}$ for $E_{\gamma}=0.1 E$.

In the collision frame, the energy of a pion, generated sufficiently above the threshold, is $\epsilon_{\pi}^{\prime} \simeq \epsilon_{p}^{\prime} \simeq \epsilon_{\gamma}^{\prime} \simeq \gamma \epsilon_{\gamma}(1-\cos \theta)$, where $\gamma$ is the Lorentz factor of the proton. The emitted neutrinos have an energy which is a fraction $\alpha_{0}(\simeq 5 \%)$ of the pion energy. Similarly to the statistical treatment of the pp-collisions, we write a simplified (with delta approximation instead of a function smoothed by angle averaging) kinetic equation for the isotropic distribution of the neutrinos generated by $\mathrm{p} \gamma$-collisions in the co-moving frame, which is :

$$
\frac{\partial}{\partial t} f_{\nu}+c \vec{n} . \nabla f_{\nu}=\xi_{\nu} c \sigma_{p \gamma} \int_{\gamma>\gamma_{t h}} f_{\star}(\gamma) d \gamma \int f_{\gamma}\left(\epsilon_{\gamma}\right) \delta\left(\epsilon_{\nu}-\alpha_{0} \gamma^{2} \epsilon_{\gamma}\right) d \epsilon_{\gamma} .
$$

Integrating both sides of the equation over the co-volume and the proper time, we easily get the number of emitted neutrinos :

$$
N_{\nu}=\xi_{\nu} N_{\star}^{>}=\xi_{\nu} N_{\star}\left(\frac{1}{\gamma_{t h}}-\frac{1}{\gamma_{\max }}\right)
$$

where $N_{\star}^{>}$is the number of cosmic rays above the p $\gamma$-threshold energy, the last result being obtained for a $\gamma^{-2}$ proton distribution. The integration, like 
in the case of pp-collisions, starts at the p $\gamma$-"proto"-sphere. The total energy radiated by neutrinos in the co-moving flow is also derived easily from the kinetic equation :

$$
E_{\nu}^{\prime} \sim \alpha_{0} \xi_{\nu} N_{*} \log \left(\frac{\gamma_{\max }}{\gamma_{t h}}\right) \bar{\epsilon}_{\gamma}^{\prime}
$$

The logarithm factor is obtained for a $\gamma^{-2}$ distribution of protons and can easily be modified. This result can also be rewritten in the observer frame as follows :

$$
E_{\nu} \sim \alpha_{0} \gamma_{t h} \bar{\epsilon}_{\gamma} \log \left(\frac{\gamma_{\max }}{\gamma_{t h}}\right) N_{\nu}
$$

The energy radiated in the form of $\mathrm{p} \gamma$-neutrinos is $E_{\nu} \sim 10^{-7} E$. Numerical computation of such spectra has been done (see Mücke et al. (1998)). The kinetic equation also provides with the neutrino spectrum. Assuming $f_{\star}$ is a power law distribution and that the energy distribution of target photons is $\epsilon_{\gamma} f_{\gamma} \propto \epsilon_{\gamma}^{-\alpha}$, we get a power law energy spectrum for the neutrinos, namely

$$
\epsilon_{\nu}^{2} \frac{d N_{\nu}\left(>\epsilon_{\nu}\right)}{d \epsilon_{\nu}} \propto \epsilon_{\nu}^{-\frac{s-1}{2}}
$$

in an energy range depending on the energy range of the protons since $\epsilon_{\nu} \simeq$ $\alpha_{0} \gamma^{2} \epsilon_{\gamma}$. These spectra are given in the co-moving frame. For the observer, they are Doppler beamed with the bulk Lorentz factor of the relativistic wind. However, the number of events is so low that it is still "virtual" to talk about a spectrum...

\section{Discussion}

In order to account for the non-thermal and highly variable gamma emission of GRBs, the fireball model and the internal shock model have been designed with baryonic load parameter $\eta$ that has been supposed large enough to get a relativistic wind achieving a large Lorentz factor $\Gamma \sim \eta$. However, it turns out that opacity effects could easily be significant at the beginning of the emission. We stress that point through a discussion involving a critical value $\eta_{\star}$ of the baryon load parameter. Indeed one emphasis of this paper is to analyse the opacity of the GRB to relativistic protons with respect to pion production by pp-collisions. Actually, the opacity condition relative to pp-collision is not far from the Compton opacity condition. Therefore it is 
reasonable to think that a significant fraction of the GRBs experience proton Fermi acceleration with efficient pp-collisions revealed by neutrino radiation.

During a stage of pp-opacity, we have shown that Fermi acceleration leads to conclusions about the pp-process that are very sensitive to the choice of the efficiency law of the acceleration. We have shown that the Bohm scaling assumption leads to an efficient proton acceleration that would be limited by synchroton loss, whereas the correct law governed by the turbulence spectrum (Kolmogorov law was used) leads to very different estimates and the pp-collisions process turns out to be the main limitation of the proton energy. Moreover, the excessive efficiency of the Bohm scaling would make the acceleration to deplete its energy reservoir in a time too short to maintain the gamma emission. The most important surprise raised in estimating the energy limitation in the radiation free stage. Indeed, the expansion limitation turns out to be drastic with the Kolmogorov law and maintains the proton energy below a few $10^{5} \mathrm{GeV}$.

The analysis of the paper indicates that a double neutrino emission can be expected with many GRBs, namely a stage of pp-neutrino emission followed by a stage of $\mathrm{p} \gamma$-neutrinos. The number of emitted pp-neutrinos gives the amount of relativistic protons; and the number of $\mathrm{p} \gamma$-neutrinos gives the number of protons above the threshold, which is about $10^{-4}$ less. We proposed an analytical shape of the neutrinos spectra, as well as the photon spectrum generated by the $\pi^{0}$-decay.

The magnetic field intensity is an important parameter that controls the proton acceleration and the synchrotron losses. The neutrino emissions significatively depend on its value at the crucial distance $r_{b}$. We took a high but still reasonable value of $10^{7} G$ at this distance; if we take less, the synchrotron limitation is less important, but the expansion limitation, which controls the highest energy of the protons in the GRB, becomes more severe, and also the pp-neutrino emission becomes less energetic. If we unduly take more, synchrotron losses dominate over pp-collisions and the acceleration is more efficient against the expansion losses, however not sufficient to get UHE-cosmic rays... This paper does not exclude the possibility of UHEcosmic ray generation in GRBs. It simply states that its achievement with Bohm scaling is not reliable and leads to observational inconsistencies and that its achievement with Kolmogorov scaling is impossible... We think that there is another possibility (Pelletier (1999), Pelletier \& Kersalé (2000)) that deserves a detailed investigation that we will present in a forthcoming paper. 
Acknowledgement : The authors are grateful to Frédéric Daigne, Gilles Henri, Martin Lemoine and Eli Waxman for fruitful discussions.

\section{References}

Beloborodov A. M., Stern B. E., Svensson R., 2000, ApJ, 535, 158

Casse F., Lemoine M., Pelletier G., 2001, Phys. Rev. D, 65, 3002

Daigne F., Mochkovitch R., 1998, MNRAS, 296, 275

Derishev E. V., Kocharovsky V. V., Kocharovsky V. V., 1999, ApJ, 521, 640

Dermer C. D., 2001, Ultra-high Energy Cosmic Rays and Neutron-Decay Halos from Gamma Ray Bursts, in Gamma-ray Bursts in the Afterglow Era, p. 269

Fishman G. J., Meegan C. A., 1995, ARA\&A, 33, 415

Goodman J., 1986, ApJ, 308, L47

Lemoine M., 2002, A \& A, 390, L31

Mészáros P., 2002, ARA\&A, 40, 137

Mészáros P., Laguna P., Rees M. J., 1993, ApJ, 415, 181

Mücke A., Engel R. R., Protheroe R. J., Rachen J. P., Stanev T., 1998, Photomeson production in astrophysical environments, in Abstracts of the 19th Texas Symposium on Relativistic Astrophysics and Cosmology, held in Paris, France, Dec. 14-18, 1998. Eds.: J. Paul, T. Montmerle, and E. Aubourg (CEA Saclay).

Paczyński B., Xu G., 1994, ApJ, 427, 708

Pelletier G., 1999, A \& A, 350, 705

Pelletier G., Kersalé E., 2000, A \& A, 361, 788

Rees M. J., Mészáros P., 1992, MNRAS, 258, 41P

Rees M. J., Mészáros P., 1994, ApJL, 430, L93 
Vietri M., 1995, ApJ, 453, 883

Waxman E., 1995, Phys. Rev. Lett., 75, 386

Waxman E., Bahcall J., 1999, Phys. Rev. D, 59 